\title{
Mesoporous, microporous and nanowired: electron microscopy of aerogel composites
}

\author{
Rhonda M. Stroud*, Jeffrey W. Long**, Jeremy J. Pietron**, Erik M. Lucas**, Michele L. \\ Anderson***, Karen E. Swider Lyons**, Celia I. Merzbacher**** and Debra R. Rolison** \\ *Naval Research Laboratory, Code 6371, 4555 Overlook Avenue SW, Washington, DC 20375 \\ **Naval Research Laboratory, Code 6171, 4555 Overlook Avenue SW, Washington, DC 20375 \\ ***Naval Surface Warfare Center, 9500 MacArthur Boulevard, West Bethesda, MD 20817 \\ ****Naval Research Laboratory, Code 1004, 4555 Overlook Avenue SW, Washington, DC 20375
}

Sol-gel synthesis is an extremely flexible technique for producing porous, low-density nanocomposite materials [1,2]. Metal or other nanoparticles can be incorporated into an about-to-gel sol; the sol is then gelled and dried, trapping the suspended nanoparticles. If the gel is supercritically dried, wherein the pore liquid is extracted above the critical point, an aerogel, with a connected network of mesopores, is obtained (Fig. 1a). The electrical conductivity, optical absorption and chemical activity of these composites can be tuned by simply altering the composition and concentration of nanoparticles guests prior to incorporation in the gel network. Furthermore, because of the connected mesopore network, it is possible to chemically address and modify both the aerogel surfaces and incorporated nanoparticles in situ (Fig. 1b) [3]. Diverse aerogel nanocomposites including Au-silica, Au-titania, Pt-silica, carbon black-silica, zeolite-silica, and titania-silica, have been produced in this manner.

Important questions for the development of aerogel nanocomposites include how the nanoparticles are incorporated into the gel host, and whether the presence of the nanoparticles affects the gel structure. Traditionally, the structure of aerogels is studied through a combination of gas physisorption measurements [4], to characterize surface area, pore volume and pore size distribution, and small-angle neutron scattering (SANS) [5], to determine the distribution of gel particle sizes and shapes. Limited transmission electron microscopy (TEM) work has been done on aerogels, [6] due to their insulating properties and electron beam sensitivity. Despite these difficulties, TEM remains the best technique for characterization of the local structural relationship between the aerogel matrix and guest nanoparticles (Fig. 2). By comparison of SANS, physisorption and TEM studies we have demonstrated that for the noble-metal nanoparticle-silica aerogel composites there is no significant alteration of the pore architecture due to the incorporation of nanoparticles ranging in diameter from 2 to $100 \mathrm{~nm}[7]$.

For catalytic, electrocatalytic and chemical sensing applications, a critical feature of the nanocomposites is the chemically accessible surface of the guest nanoparticles. The nanoparticles must be mechanically stable inside the gel, and the gel nanocomposites must be able to withstand liquid immersion. However, the nanoparticles cannot be so encased in gel as to be screened from the pore network. The challenge is to determine what combination of nanoparticle size, sol chemistry and drying method produces the optimum mechanical stability and accessible nanoparticle surface. Our results show that for particles $5 \mathrm{~nm}$ and larger, the particle surfaces remain chemically accessible in noble metal-silica aerogel composites. Smaller particles, e.g. 2-nm Pt, are often completely encased by the silica aerogel matrix. The encasement problem can be overcome by impregnating traditional metal-on-carbon catalysts with silica gel (Fig. 3). Because the silica gel provides a 
mesoporous pathway to the carbon-supported Pt sites, while minimizing the agglomeration of the catalyst particles, dramatic improvements in catalytic activity can be achieved [8].

\section{References}

[1] C.A. Morris et al. Science 284 (1999) 622.

[2] M.L. Anderson et al. Advanced Engineering Materials 2 (8) (2000) 481.

[3] J.V. Ryan et al. Nature 406 (2000) 169.

[4] G. Schuck et al. in Aerogels (Ed.: J. Fricke), Springer-Verlag, Berlin, 1996; M.-A. Einarsrud et al. in Chemical Processing of Advanced Materials (Eds.: L.L. Hench, J.K. West) John Wiley, New York, 1992; C.J. Brinker, G.W. Scherer Sol-Gel Science, Academic Press, New York, 1990.

[5] D.W. Schaefer, K.D. Keefer Phys. Rev. Lett. 56 (1986) 2199; A. Emmerling, et al. J. Non-Cryst. Solids 125 (1999) 230.

[6] G.C. Ruben et al. J. Non-Cryst. Solids 186 (1995) 209.

[7] M.L. Anderson et al. Langmuir 15 (1999) 674.

[8] M.L. Anderson et al.Nanoletters, in the press.

[9] This work was partially supported by the Office of Naval Research and Defense Advanced Research Projects Agency.

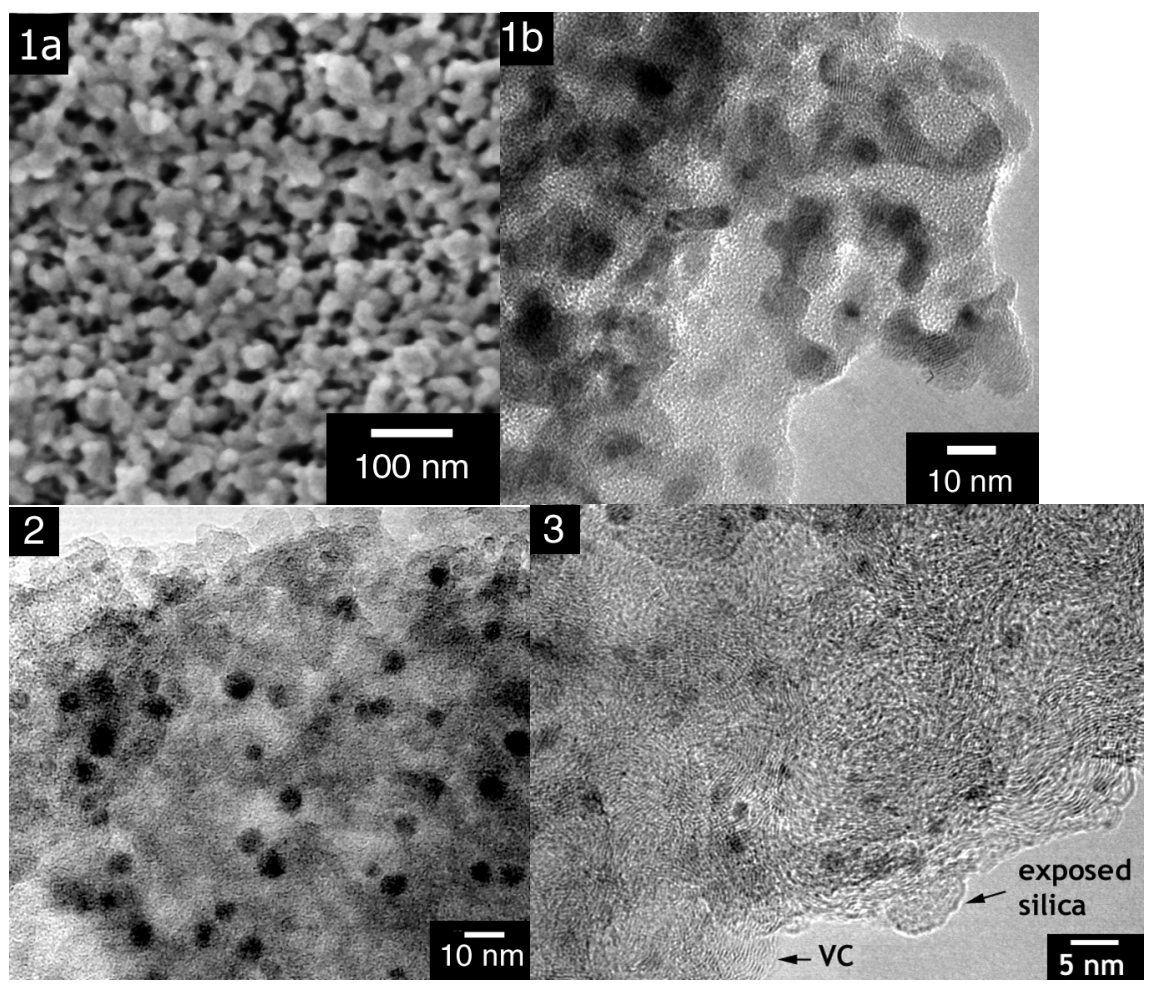

FIG. 1 (a) SEM micrograph of a 100-nm Au-silica aerogel nanocomposite, showing the mesoporous network (black). (b) TEM image of crystalline $\mathrm{RuO}_{2}$ nanowires grown in situ on an amorphoussilica aerogel host.

FIG. 2 TEM image showing the distribution of 5-nm metal particles in a Au-silica aerogel composite.

FIG. 3 TEM image of a Pt-modified Vulcan carbon-silica composite. 